\title{
AN INVERSE PROBLEM OF THE CALCULUS OF VARIATIONS FOR MULTIPLE INTEGRALS*
}

\author{
BY \\ LINCOLN LA PAZ $\dagger$ \\ 1. INTRODUCTION
}

Hirsch $\ddagger$ has proved that if the equation of variation of the second-order partial differential equation

(1.1) $F(x, y, z, p, q, r, s, t)=0, z=z(x, y), p=z_{x}, \cdots, t=z_{y y}$,

is self-adjoint, then the equation (1.1) has the form

$$
M\left(r t-s^{2}\right)+R r+2 S s+T t+N=0,
$$

where $M, R, S, T, N$ are functions of $x, y, z, p, q$ alone; and he has shown that in this case there exists a function $f$, itself of the second order, of the form $\S$

$$
f=g(x, y, z, p, q) s+c(x, y, z, p, q),
$$

such that the equation (1.2) is the Lagrange partial differential equation of a problem of minimizing the double integral

$$
J=\iint f(x, y, z, p, q, s) d x d y .
$$

A function $f$ thus related to a given partial differential equation with self-adjoint equation of variation will for brevity be referred to as associated with the given equation.

Kürschák, đ utilizing the method of Hirsch, extended the latter's theorem to partial differential equations of the form

$$
\begin{gathered}
F\left(x_{1}, \cdots, x_{n}, z, p_{1}, \cdots, p_{n}, p_{11}, p_{12}, \cdots, p_{n n}\right)=0, \\
p_{i}=\partial z / \partial x_{i}, \quad p_{i j}=\partial^{2} z / \partial x_{i} \partial x_{j} .
\end{gathered}
$$

Neither Hirsch nor Kürschák attempted to find either the most general

* Presented to the Society, August 29,1929, and February 22, 1930; received by the editors January 14, 1930.

$\dagger$ National Research Fellow in Mathematics, 1928-1929.

$\ddagger$ A. Hirsch, Mathematische Annalen, vol. 49 (1897), p. 49. Loc. cit., §9.

$\S$ The functions $g$ and $c$ in (1.3) must satisfy certain partial differential equations of the second order involving the coefficients of the equation (1.2).

ๆ J. Kürschák, Mathematische Annalen, vol. 60 (1905), p: 157. 
function of the second order associated with a given equation or the most general first-order function associated with a given equation linear (necessarily) in the second partial derivatives. Koenigsberger,* without the use of the self-adjoint theory, obtained a formula for the integrand function $f$ of a problem

$$
I=\iint f(x, y, z, p, q) d x d y,
$$

having a given partial differential equation (1.1), linear in $r, s, t$, as its Lagrange equation; but his formula does not give the most general such function.

The determination of the most general second-order function associated with a given equation (1.5) is rendered irregular by the fact that an initial restriction must be placed on the form of such a function in order to insure the degeneration of the corresponding Lagrange partial differential equation from the fourth to the second order. This case is not treated in the present paper which is devoted to a determination of the most general first-order integrand function $f\left(x_{1}, \ldots, x_{n}, z, p_{1}, \ldots, p_{n}\right)$ associated with a partial differential equation (1.5) linear in the $p_{i j}$. The argument of $\$ 3$ leading to this determination is based on the self-adjoint theory and is in part similar to that employed by Davis $\dagger$ in connection with an analogous question for simple integrals

$$
I=\int_{x_{1}}^{x_{2}} f\left(x, y, z, y^{\prime}, z^{\prime}\right) d x,
$$

having as their Euler equations a given pair of ordinary differential equations with self-adjoint equations of variation.

In the last two sections application of the results obtained is made to secure a generalization of Dirichlet's principle and to treat the problem of plane geometries in which straight lines are shortest.

\section{Properties of the Lagrange equation}

For a problem of minimizing the multiple integral

$$
I=\int_{(n)} f\left(x_{1}, \cdots, x_{n}, z, p_{1}, \cdots, p_{n}\right) d x_{1} \cdots d x_{n},
$$

the Lagrange partial differential equation has the form

$$
f_{p_{a} x_{a}}+f_{p_{a}{ }_{a} p_{a}}+f_{p_{a} p_{b} p_{a b}}-f_{z}=0 .
$$

* L. Koenigsberger, Sitzungsberichte der Akademie der Wissenschaften, Berlin, 1905, p. 250.

† D. R. Davis, these Transactions, vol. 30 (1928), p. 717. 
Here, as elsewhere in this paper, the repeated subscripts $a$ and $b$ are umbral indices with the ranges 1 to $n$.

Consider a one-parameter family of integral surfaces, $z=z\left(x_{1}, \ldots, x_{n}, k\right)$, of the partial differential equation (2.2). The quantity $u$ defined by $u=\partial z\left(x_{1}, \ldots, x_{n}, k\right) / \partial k$ is called the variation of the family on the surface corresponding to the value $k$ of the parameter. On this surface $u$ satisfies the equation of variation of (2.2) which can be put in the form

$$
A(u)=\left(\partial f_{p_{a}^{z}} / \partial x_{a}-f_{z z}\right) u+\left(\partial f_{p_{a} p_{b}} / \partial x_{a}\right) u_{b}+f_{p_{a} p_{b}} u_{a b}=0,
$$

where the differentiations with respect to the $x_{i}$ are partial when $z$ and its derivatives are considered as functions of the $x$ 's, and where $u_{b}=\partial u / \partial x_{b}$, $u_{a b}=\partial^{2} u / \partial x_{a} \partial x_{b}$. From (2.3) it can be shown that $A(u)$ and its adjoint $A^{\prime}(u)^{*}$ are identical.

The Lagrange partial differential equation of a problem (2.1) is therefore an equation of the form

$$
A_{a b} p_{a b}+B=0 \text {, }
$$

with an equation of variation which is self-adjoint on every hypersurface $z=z\left(x_{1}, \cdots, x_{n}\right)$. In (2.4), $A_{i j}, B$ are functions of the variables $x_{i}, z, p_{i}$ alone and $A_{i j}=A_{j i}$.

A first necessary condition for the equation of variation of a partial differential equation (1.5) to be self-adjoint is that $F$ satisfy the relations $\dagger$

$$
2 \partial F / \partial p_{i}-\sum_{k=1}^{n}\left(\partial / \partial x_{k}\right)\left(\partial F / \partial p_{i k}\right)\left(1+\delta_{k}^{i}\right)=0 \quad(i=1, \cdots, n),
$$

where $\delta_{k}^{i}$ is the Kronecker delta. When $F$ has the special form (2.4) these conditions become

(2.5) $\left(A_{i b}\right)_{x_{b}}+\left(A_{i b}\right)_{z} p_{b}+\left\{\left(A_{i b}\right)_{p_{a}}-\left(A_{a b}\right)_{p_{i}}\right\} p_{a b}-B_{p_{i}}=0(i=1, \cdots, n)$.

Hence, since these relations are required to hold on every hypersurface $z=z\left(x_{1}, \ldots, x_{n}\right)$, we have

* The notation is that of Frobenius, Crelle's Journal, vol. 85, p. 207. The actual computation of $A^{\prime}(u)$ can be avoided by the following artifice similar to one used by Hadamard (Lecons sur le Calcul des Variations, p. 319) in connection with ordinary linear differential expressions of the second order. Introduce a quantity $w$ defined by the equation

then it can be shown that

$$
2 w=f_{z z} u^{2}+2 f_{s p_{a}} u u_{a}+f_{p_{a p b}} u_{a} u_{b} ;
$$

$$
u A(v)-v A(u)=\partial\left(u \partial w / \partial v_{a}^{\prime}-v \partial w / \partial u_{a}^{\prime}\right) / \partial x_{a} .
$$

$\dagger$ Kürschák, loc. cit., p. 160. The writer has found that the conditions $(K)$ are also sufficient conditions for self-adjointness. 
TheOREM I. An equation (1.5) which as it stands is to be the Lagrange partial differential equation of a problem (2.1) must necessarily be of the form (2.4) with coefficients $A_{i j}, B$ satisfying the conditions

$$
\begin{array}{r}
A_{i j}=A_{j i}, \quad\left(A_{i j}\right)_{p_{k}}=\left(A_{k j}\right)_{p_{i}} \quad \begin{array}{r}
(i, j, k=1, \cdots, n), \quad k \neq i, \\
\left(A_{i b}\right)_{x_{b}}+\left(A_{i b}\right)_{z} p_{b}-B_{p_{i}}=0
\end{array} \quad(i=1, \cdots, n),
\end{array}
$$

identically in the variables $x_{i}, z, p_{i}$.

\section{DETERMINATION OF THE MOST GENERAL FIRST-ORDER INTEGRAND FUNCTION}

Suppose that an equation (2.4) with coefficients satisfying the conditions (2.6) is given. In view of the relations in the first row of (2.6) we can define a function $g\left(x_{1}, \ldots, x_{n}, z, p_{1}, \ldots, p_{n}\right)$ by means of the system of equations

$$
g_{p_{i} p_{j}}=A_{i j} \quad(i, j=1,2, \cdots, n),
$$

and the function $g$ thus defined can be expressed as a line integral

$$
g=\int_{0}^{p} H_{a} d p_{a}
$$

where

$$
H_{i}=\int_{0}^{p} A_{i b} d p_{b} \quad(i=1,2, \cdots, n) .
$$

In (3.2) and (3.3) the symbols 0 and $p$, used as limits, represent $n$-partite variables. It is clear that if $G$ is a particular solution of (3.1) then the most general solution of this system is of the form

$$
\begin{aligned}
g=G\left(x_{1}, \cdots, x_{n}, z, p_{1},\right. & \left.\cdots, p_{n}\right) \\
& +C\left(x_{1}, \cdots, x_{n}, z\right)+D_{a}\left(x_{1}, \cdots, x_{n}, z\right) p_{a} .
\end{aligned}
$$

If, now, the given equation (2.4) is to be the Lagrange equation of a problem (2.1) formed with the function $g$ in (3.4) as the integrand of the multiple integral, then the following relation must hold identically in the variables $x_{i}, z, p_{i}, p_{i k}$ :

$$
\partial g_{p_{a}} / \partial x_{a}-g_{z}=A_{a b} p_{a b}+B .
$$

Reduction of (3.5) by means of (3.1) shows that the relation below must hold identically in the variables $x_{i}, z, p_{i}$ :

$$
C_{z}-D_{a x a}=G_{p_{a} x}+G_{p_{a} z} p_{a}-G_{z}-B .
$$

If there are to exist functions $C, D_{i}(i=1, \ldots, n)$ of $x_{1}, \ldots, x_{n}$ and $z$ alone 
satisfying (3.6), then the right member of this equation must be independent of $p_{i}(i=1, \ldots, n)$. That this condition is actually fulfilled is seen by differentiating the right side of (3.6) with respect to $p_{i}$ and referring to the set of relations in the second row of (2.6).

Solutions of (3.6) for $C, D_{i}$ as functions of $x_{1}, \ldots, x_{n}, z$ alone therefore always exist, and if the set $\left(C, D_{i}\right)$ gives a particular solution of this equation then the most general solution is given by the set $\left(C+c, D_{i}+d_{i}\right)$ where $\left(c, d_{i}\right)$ are functions of $x_{1}, \ldots, \hat{x}_{n}, z$ satisfying the equation

$$
c_{z}-d_{a x_{a}}=0 \text {, }
$$

but otherwise arbitrary.

The relation (3.7) is a necessary and sufficient condition for the Lagrange partial differential equation of a problem of minimizing the multiple integral

$$
\int_{(n)}\left(c+d_{a} p_{a}\right) d x_{1} \cdots d x_{n}
$$

to be identically satisfied. From this it follows, in view of a theorem of Koenigsberger* on the conditions under which the Lagrange equation can be identically satisfied, that the quantity $c+d_{a} p_{a}$ is representable as a sum of partial $\dagger$ derivatives with respect to the $x_{i}$ of arbitrary functions $w_{i}$ of $x_{1}, \ldots, x_{n}, z$ alone

$$
c+d_{a} p_{a}=\partial w_{1} / \partial x_{1}+\cdots+. \partial w_{n} / \partial x_{n} .
$$

This completes the proof of

Theorem II. If an equation $F\left(x_{1}, \cdots, x_{n}, z, p_{1}, \cdots, p_{n}, p_{11}, \cdots, p_{n n}\right)=0$ is linear in the $p_{i j}$ and has an equation of variation which is self-adjoint on every hypersurface $z=z\left(x_{1}, \ldots, x_{n}\right)$ then there is always a multiple integral of the form

$$
I=\int_{(n)} f\left(x_{1}, \cdots, x_{n}, z, p_{1}, \cdots, p_{n}\right) d x_{1} \cdots d x_{n}
$$

having $F=0$ as its Lagrange partial differential equation. The most general such integral has an integrand function of the form

$$
f=G+C+D_{a} p_{a}+\partial w_{1} / \partial x_{1}+\cdots+\partial w_{n} / \partial x_{n},
$$

where $G\left(x_{1}, \ldots, x_{n}, z, p_{1}, \cdots, p_{n}\right)$ is a particular solution of (3.1), where the set $\left\{C\left(x_{1}, \ldots, x_{n}, z\right), D_{i}\left(x_{1}, \ldots, x_{n}, z\right)\right\}$ is a particular solution of (3.6)and where the $w_{i}$ occurring in the partial derivatives with respect to $x_{i}$ are arbitrary functions of $x_{1}, \cdots, x_{n}, z$ alone.

* Koenigsberger, Mathematische Annalen, vol. 62, p. 123.

$\dagger$ When $z$ is regarded as a function of the $x$ 's. 
As an immediate corollary we have a theorem analogous to one stated by Hirsch for simple integrals:*

THEOREM III. If the Lagrange partial differential equation of a problem of minimizing a multiple integral with second-order integrand function

$$
\int_{(n)} f_{2}\left(x_{1}, \cdots, x_{n}, z, p_{1}, \cdots, p_{n}, p_{11}, \cdots, p_{n n}\right) d x_{1} \cdots d x_{n}
$$

degenerates into a partial differential equation, $L=0$, of the second order and linear in the $p_{i j}$, then by means of quadratures a first-order integrand function $f\left(x_{1}, \ldots, x_{n}, z, p_{1}, \ldots, p_{n}\right)$ can be determined such that a problem of minimizing the integral

$$
\int_{(n)} f_{1}\left(x_{1}, \cdots, x_{n}, z, p_{1}, \cdots, p_{n}\right) d x_{1} \cdots d x_{n}
$$

leads to the same partial differential equation, $L=0$.

\section{A geNeralization of Dirichlet's PRINCIPLE}

The partial differential equation

$$
F=\partial^{2} z / \partial x_{1}^{2}+\partial^{2} z / \partial x_{2}^{2}+\cdots+\partial^{2} z / \partial x_{n}^{2}=0
$$

satisfies the conditions of Theorem II of the last section. The system (3.1) is in this case

$$
g_{p_{i} p_{j}}=\delta_{j}^{i} .
$$

Hence we may take for the $G$ function in (3.10)

$$
G=\frac{1}{2}\left(p_{1}^{2}+\cdots+p_{n}^{2}\right) .
$$

By means of (4.1) the relation (3.6) reduces to

$$
C_{z}-D_{a x a}=0 \text {. }
$$

Hence the sum $C+D_{a} p_{a}$ in (3.10) can itself be represented in the form (3.9). We conclude that the most general $n$-fold integral which has (4.1) as it stands as its Lagrange equation has an integrand function of the form

$$
f=\frac{1}{2}\left(p_{1}{ }^{2}+\cdots+p_{n}{ }^{2}+\partial W_{1} / \partial x_{1}+\cdots+\partial W_{n} / \partial x_{n}\right),
$$

where the functions $W_{i}$ are arbitrary functions of $x_{1}, \cdots, x_{n}$ and $z$.

The expression in parentheses can be written in the form

(4.6) $\mathfrak{F}\left(x_{1}, \cdots, x_{n}, z, p_{1}, \cdots, p_{n}\right)=\sum_{i=1}^{n}\left(p_{i}+W_{i z} / 2\right)^{2}+\left(W_{i x_{i}}-W_{i z /}^{2} / 4\right)$.

\footnotetext{
${ }^{*}$ Hirsch, loc. cit., pp. 52-53.
} 
A generalization of Dirichlet's principle would now assert that if the function $\mathfrak{F}$ is never negative in a region $S$ which consists of all sets of values $\left(x_{1}, \cdots, x_{n}, z, p_{1}, \cdots, p_{n}\right)$ where $\left(x_{1}, \cdots, x_{n}, z\right)$ is a point in a certain domain $D$ of $\left(x_{1}, \cdots, x_{n}, z\right)$-space and where $p_{1}, \ldots, p_{n}$ have arbitrary finite values, then there actually exists a hypersurface $z=z\left(x_{1}, \ldots, x_{n}\right)$ minimizing the $n$-fold integral

$$
\mathfrak{D}=\int_{(n)} \mathfrak{F} d x_{1} \cdots d x_{n},
$$

at least in $D$.

For $n=2$ the partial differential equation (4.1) becomes the Laplacian, and there is thus established an interesting relation between the class of analytic functions of a complex variable and the class of real functions $z(x, y)$ which furnish solutions of a problem of minimizing double integrals of the form

$$
\iint\left(p^{2}+q^{2}+\partial W_{1} / \partial x+\partial W_{2} / \partial y\right) d x d y .
$$

The author hopes to investigate in a later paper the corresponding relationships for problems of the calculus of variations having as their Lagrange equation the generalized Laplace equation by means of which Hedrick and others have classified functions in the plane.*

\section{GeOMETRIES IN WHICH STRAIGHT LINES ARE THE CURVES OF SHORTEST LENGTH}

As a further application of the results obtained in Theorems I and II, we consider the problem, first treated by Hamel in his Dissertation, of determining the most general geometry in the plane for which straight lines are the curves of shortest length joining two given points. $\dagger$ It will be shown that Hamel did not obtain the most general such geometry.

Denote by $R$ the region which consists of all sets of values $\left(x, z, z^{\prime}\right)$ where $(x, z)$ is a point in the $x z$-plane and $z^{\prime}$ has an arbitrary finite value. Denote by $H$ the class of real functions $h\left(x, z, z^{\prime}\right)$ which are positive and single-valued in $R$ and which together with their partial derivatives of the first three orders are continuous in this region. $\ddagger$

Then formulated as an inverse problem of the calculus of variations Hamel's problem requires the determination of the most general function,

* Hedrick, Ingold and Westfall, Journal de Mathématiques, (9), vol. 2 (1923), p. 338, \$10.

$\dagger$ Hamel, Mathematische Annalen, vol. 57 (1903), pp. 235-244.

$\ddagger$ Functions of class $H$ satisfy all of the conditions imposed by the geometric axioms chosen by Hamel and the definition of length adopted by him. In Bolza's terminology the functions $h\left(x, z, z^{\prime}\right)$ are of class $C^{\prime \prime \prime}$ in $R$. See Vorlesungen über Variationsrechnung, p. 13. 
$g\left(x, z, z^{\prime}\right)$, in the class $H$ such that the straight line segment $E_{12}$ joining the arbitrary but fixed points $1:\left(x_{1}, z_{1}\right)$ and $2:\left(x_{2}, z_{2}\right), x_{2} \neq x_{1}$, renders the length integral

$$
L=\int_{x_{1}}^{x_{2}} g\left(x, z, z^{\prime}\right) d x
$$

a proper, ${ }^{*}$ absolute minimum with respect to all other admissible $\dagger$ arcs, $z=z(x)$, joining. these two points.

If the integral (5.1) is to have even a strong relative minimum on the straight line segment $E_{12}$, then $E_{12}$ must satisfy the necessary conditions of Euler, Weierstrass and Legendre. $\ddagger$ Since the minimizing arcs are now prescribed these conditions must be regarded as necessary conditions on the integrand function $g\left(x, z, z^{\prime}\right)$ in (5.1). Thus the first or Euler condition is seen to require that the function $g\left(x, z, z^{\prime}\right)$ be so specialized that the twoparameter family of straight lines in the $x z$-plane is the extremal system of (5.1). Our Theorem I, written for $n=1$, shows that the most general equation $F\left(x, z, z^{\prime}, z^{\prime \prime}\right)=0$, linear in $z^{\prime \prime}$, with an equation of variation self-adjoint on every curve $z=z(x)$, which is equivalent to an equation of the form

$$
z^{\prime \prime}=G\left(x, z, z^{\prime}\right)
$$

is of the form

$$
F=P z^{\prime \prime}-P G=0,
$$

where the multiplier $P\left(x, z, z^{\prime}\right)$ of class $C^{\prime}$ is $\not \equiv 0$ and satisfies the relation

$$
P_{x}+P_{z} z^{\prime}+P_{z^{\prime}} G+P G_{z^{\prime}}=0 \text {. }
$$

The straight lines in question satisfy (5.2) with $G=0$. The most general solution of (5.4), when $G=0$, is $P=P\left(z^{\prime}, z-x z^{\prime}\right)$, where $P$, except for the restrictions just mentioned, is an arbitrary function of its arguments. Hence our problem requires the determination of the most general integrand function $g\left(x, z, z^{\prime}\right)$ such that the Euler equation of (5.1) is

$$
P\left(z^{\prime}, z-x z^{\prime}\right) z^{\prime \prime}=0 .
$$

From Theorem II with $n=1$ the most general such function is seen to be

$$
g=C\left(x, z, z^{\prime}\right)+D(x, z)+E(x, z) z^{\prime}+d u(x, z) / d x,
$$

\footnotetext{
* Compare Hilbert, Bulletin of the American Mathematical Society, vol. 8 (1902), p. 450.

$\dagger$ For the meaning of this term and the significance of the region $R$ introduced above, see Bliss, Calculus of Variations, 1925, pp. 128-129.

$\ddagger$ Bliss, loc. cit., pp. 130-132. The field condition is automatically satisfied since the limits of integration are fixed and the one-parameter family of straight lines through a point has no second envelope.
} 
where $C$ is a particular solution of

$$
C_{z^{\prime} z^{\prime}}=P\left(z^{\prime}, z-x z^{\prime}\right),
$$

where the set $(D, E)$ is a particular solution of

$$
D_{z}-E_{x}=C_{z^{\prime} x}+C_{z^{\prime} z} z^{\prime}-C_{z},
$$

and where $u$ is an arbitrary function of $x$ and $z$. The value for $C$ determined from (5.7) and the relation (5.4) written for $G=0$, enable us to show that the right side of (5.8) is identically zero. Therefore $D+z^{\prime} E$ is itself the derivative of an arbitrary function of $x$ and $z$. Hence

$$
g\left(x, z, z^{\prime}\right)=\int_{z_{0}^{\prime}}^{z^{\prime}} \int_{z_{0}^{\prime}}^{z^{\prime}} P\left(z^{\prime}, z-x z^{\prime}\right) d z^{\prime} d z^{\prime}+d w / d x,
$$

where it is understood that the arbitrary elements $z_{0}^{\prime}, P\left(z^{\prime}, z-x z^{\prime}\right)$ and $w(x, z)$ are to be so chosen that $g$ is a member of the class $H .^{*}$

For functions $P\left(z^{\prime}, z-x z^{\prime}\right)$ such that the vanishing of $P$ implies the relation $z^{\prime \prime}=0$, the argument now proceeds in the original region $R$; for functions not of this character the argument is made in the largest subregion $R_{1}$ of $R$ in which $P \neq 0$ and in which the sets $\left(x, z, z^{\prime}\right)$ with $z_{1}^{\prime}<z^{\prime}<z_{2}^{\prime}$ are admissible if the sets $\left(x, z, z_{1}^{\prime}\right)$ and $\left(x, z, z_{2}^{\prime}\right)$ possess this property. The conditions of Weierstrass and Legendre, since they must hold for arbitrary line segments $E_{12}$, require respectively

$$
E\left(x, z, z^{\prime}, Z^{\prime}\right) \geqq 0,
$$

at every admissible element $\dagger\left(x, z, z^{\prime}\right)$, for every admissible element $\left(x, z, Z^{\prime}\right) \neq\left(x, z, z^{\prime}\right)$, and

$$
g_{z^{\prime} z^{\prime}} \geqq 0,
$$

at every admissible element $\left(x, z, z^{\prime}\right)$. In view of the nature of the regions $R$ and $R_{1}$, it is clear that we may in every case employ the relation $\ddagger$

$$
E\left(x, z, z^{\prime}, Z^{\prime}\right)=\frac{1}{2}\left(Z^{\prime}-z^{\prime}\right)^{2} g_{z^{\prime} z^{\prime}}\left(x, z, z^{\prime}+\theta\left(Z^{\prime}-z^{\prime}\right)\right), 0<\theta<1,
$$

* Such an agreement is necessary since the integral (5.1) formed with an integrand $g\left(x, z, z^{\prime}\right)$ determined out of (5.9) may be minimized by the straight lines in the $x z$-plane without $g$ belonging to $H$. This fact is shown by the following example which exhibits a function $g$ not a member of $H$, for which (5.1) is minimized by the lines in question: Choose $z_{0}^{\prime}=0, P=1, w=-x z$; then from (5.9) $g=\frac{1}{2}\left[z^{\prime}\left(z^{\prime}-2 x\right)-2 z\right]$. Hence in every point on a parabola of the family $z-a=x^{2}, a>0, g$ has the value $-z$ and is therefore $<0$. Hamel, who obtained for $g\left(x, z, z^{\prime}\right)$ the same formula (5.9) by the method of Darboux, imposes no condition on $z_{0}^{\prime}, P$, or $w$ which excludes the geometry corresponding to the above example, in which arcs of the parabolas specified have a negative length.

$\dagger$ Compare second footnote p. 516.

$\ddagger$ Bliss, loc. cit., p. 139, formula (13). 
to show that the condition (5.11) implies the condition (5.10). Consequently if $g$ is so specialized that $(5: 11)$ is satisfied it will follow that the $E$-function is $\geqq 0$ in the field* $F$ consisting of the straight lines parallel to $E_{12}$ and this is sufficient to insure that $\Delta L=L\left(C_{12}\right)-L\left(E_{12}\right)>0$ on every admissible arc $C_{12} \not \equiv E_{12}$ unless the $E$-function can vanish otherwise than ordinarily in $F . \dagger$ Hence, sufficient conditions that every straight line segment $E_{12}$ in the plane with an equation of the form $z=z(x)$ shall furnish a strong, proper, relative minimum with respect to all admissible arcs $C_{12}$ joining the points 1 and 2 are that $g$ have the form (5.9) and that in the region under consideration

$$
g_{z^{\prime} z^{\prime}}=P\left(z^{\prime}, z-x z^{\prime}\right) \geqq 0,
$$

where functions $P$ for which the equality sign holds in (5.13) are to be excluded if and only if the $E$-function computed for the corresponding integrand function (5.9) can vanish for $Z^{\prime} \neq z^{\prime}$. Since every pair of points 1 and $2, x_{1} \neq x_{2}$, in the plane can be joined by one and only one straight line segment $E_{12}$, the above conditions are also sufficient $\ddagger$ conditions for (5.1) to have a proper, absolute minimum on every $E_{12}$ of the type described. We thus reach

THEOREM IV. If a geometry in which length is defined by the integral (5.1) has an arc element of the form

$$
d L=\left\{\int_{z_{0}^{\prime}}^{z^{\prime}} \int_{z_{0}^{\prime}}^{z^{\prime}} P\left(z^{\prime}, z-x z^{\prime}\right) d z^{\prime} d z^{\prime}+d w(x, z) / d x\right\} d x,
$$

where the arbitrary elements $z_{0}^{\prime}, P\left(z^{\prime}, z-x z^{\prime}\right), w(x, z)$ are such that the quantity in brackets belongs to the class $H$ and where $P$ is never negative, with the understanding that functions $P$ which vanish are to be excluded if and only if the corresponding E-function vanishes extraordinarily; then the geometry is one for which straight lines are the curves of shortest length.

A simple example now shows that Hamel did not obtain the most general geometry in which straight lines are shortest. Choose $z_{0}^{\prime}=0, P=12 z^{\prime 2}$, and $w(x, z)=x$. Then from (5.9) $g=1+z^{\prime 4}$. This function belongs to the class $H$. Furthermore it is readily verified that the corresponding $E$-function

$$
E\left(x, z, z^{\prime}, Z^{\prime}\right)=Z^{\prime 4}-z^{\prime 4}-4\left(Z^{\prime}-z^{\prime}\right) z^{\prime 3}
$$

is always $\geqq 0$ and vanishes for $Z^{\prime}=z^{\prime}$ and for no other real value of $Z^{\prime}$. Hence the geometry for which the element of arc is $d L=\left(1+z^{\prime 4}\right) d x$ is one of

\footnotetext{
* Compare Bliss, loc. cit., pp. 27-30.

† Kneser, Lehrbuch der Variationsrechnung, 1900, \$22. Bolza, Vorlesungen über Variationsrechnung, pp. 120-121. Bliss, loc. cit., p. 133, p. 153.

‡ Sets of conditions at once necessary and sufficient are exceptional in the calculus of variations.
} 
the geometries satisfying the assumptions of Hamel, since for it the function $g$ belongs to the class $H$ and since for this geometry every straight line segment $E_{12}$ in the plane with an equation $z=z(x)$ is shorter than every other admissible arc joining the points 1 and 2 . However, the geometry here described would have to be excluded if we argued incorrectly with Hamel* that a necessary condition for a minimum is that the $E$-function be always $>0$ and therefore that the condition $g_{z^{\prime} z^{\prime}}>0$ must always be satisfied. For the special geometry just described $P=g_{z^{\prime} z^{\prime}}=12 z^{\prime 2}$ and vanishes for $z^{\prime}=0$.

Untversity of Chicago, Chicago, Illinois.

* Hamel, loc. cit., p. 243. Hamel's $E$ is the negative of our $E$-function. He writes " $\cdots E$ muss stets ein negatives Vorzeichen besitzen." 